\title{
Simulation Research on Dynamic Characteristics of Hydraulic Mount
}

\author{
Yanhua Liu* (D), Xin Guan, Pingping Lu and Rui Guo
}

\begin{abstract}
At present, research on hydraulic mounts has mainly focused on the prediction of the dynamic stiffness and loss angle. Compared to the traditional finite element analysis method, the programming method can be used to analyze hydraulic mounts for a rapid and accurate understanding of the influence of the different mounting parameters on the dynamic stiffness and loss angle. The aims of this study were to investigate the nonlinear dynamic characteristics of a hydraulic mount, and to identify the parameters that affect the dynamic stiffness and loss angle using MATLAB software programs to obtain the influence curves of the parameters, so as to use suitable parameters as the basis for vibration analysis. A nonlinear mechanical model of a hydraulic mount was established according to the basic principles of fluid dynamics. The dynamic stiffness and loss angle of the dimensionless expression were proposed. A numerical calculation method for the dynamic performance evaluation index of the hydraulic mount was derived. A one-to-one correspondence was established between the structural parameters and peak frequency of the evaluation index. The accuracy and applicability of the mechanical model were verified by the test results. The results demonstrated the accuracy of the nonlinear mechanical model of the hydraulic mount, and the vehicle driving comfort was greatly improved by the optimization of the structural parameters.
\end{abstract}

Keywords: Hydraulic mount, Vibration analysis, Dynamic characteristics, Calculation method

\section{Introduction}

With the increasing demand for driving comfort, it is becoming more important to insulate the engine and road to transmit vibrations in the vehicle. The hydraulic mount is a passive absorber that is attached to the damping mechanism, which can provide varying dynamic characteristics under different driving conditions. This is an important reason for replacing the traditional rubber mount with a hydraulic mount.

At present, research on the dynamic characteristics of hydraulic mounts is not perfect and remains in the early stages. Many scholars have invested substantial effort into studying hydraulic mounts in different manners, including early experimental methods [1-4] and more recent software analysis methods. Experimental

*Correspondence: lyh730817@163.com

State Key Laboratory of Automobile Simulation and Control, Jilin University, Changchun 130022, China methods can accurately obtain the dynamic characteristics of the hydraulic mount [5-8]. However, such experiments require a large number of different physical samples $[9,10]$, which results in a long time cycle and a large amount of investment, and it is difficult to guide the design directly. Software analysis is an effective solution to this problem. At the beginning of the design, the dynamic characteristics of the hydraulic mounts are predicted according to the 3D mathematical model or key characteristic parameters. Software analysis methods can be divided into several types. The first is based on a 3D mathematical model and the USES finite element method for simulation analysis [11-14]. The advantage of this approach is that the simulated model is closest to the real part and can best guide the design, but the cycle is long and the requirement for computer hardware is high. The second is the use of AMESIM or ADAMS software, which offers the advantage that it can better consider the motion of liquid in the simulation process, but 
the calculation cycle is relatively long [15]. The third is the use of MATLAB software [16-20] or other software [21-24] to establish a parametric model of the hydraulic mount, which can rapidly predict the dynamic characteristics according to parameter changes. Moreover, the ISIGHT software can be easily integrated for optimization design.

Based on the central parameter model, the central parameter of the structural parameters relating to the structure was optimized in this study. Specifically, through the establishment of a mechanical model, theoretical derivation, and evolution, the dynamic stiffness and loss angle of the dimensionless expressions were innovatively proposed. The model had a very good effect on the control of key performance indicators and the curves exhibited fast convergence. The accuracy of the theory was verified experimentally. Through structural parameter sensitivity analysis, the correspondence between the structural parameters and peak frequency was established, and the performance of the hydraulic mount was optimized for a certain project.

\section{Mechanical Model of Hydraulic Mount}

Based on the structure of the hydraulic mount, analysis and simplicity are necessary. The mechanical model was obtained when the upper end of the hydraulic cavity was stimulated in the vertical direction, as illustrated in Figure 1.

In Figure $1, c_{s}$ is the main spring damping of the hydraulic mount, $k_{s}$ is the main spring stiffness of the hydraulic mount, $\kappa$ is the volume flexibility of the upper chamber, $A$ is the equivalent piston area, $A_{k}$ is the crosssectional area of the inertial channel, $z_{k}$ is the displacement of fluid motion, $p_{0}$ is the upper chamber pressure, and $p_{u}$ is the lower chamber pressure.

When the upper end of the hydraulic mount is subjected to vibration from the power train, the response equation of

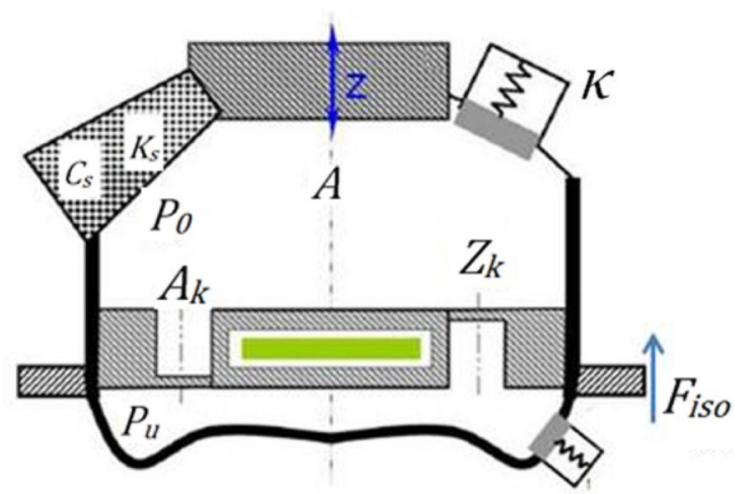

Figure 1 Schematics of hydraulic mount mechanical model the overhanging body can be obtained using the damping characteristics of the hydraulic suspension.

$$
F_{i s o}=k_{s} \cdot z+c_{s} \cdot \dot{z}+A\left(p-p_{u}\right) .
$$

According to the Laplace transform, the following equation is obtained:

$$
\frac{F_{i s o}}{z}=k_{s}+c_{s} \cdot s+A \frac{\left(p-p_{u}\right)}{z} .
$$

When the pressure changes in the upper fluid, the volume change equation is expressed as follows:

$$
\begin{aligned}
& \dot{V}=\dot{z} \cdot A=\dot{z}_{k} \cdot A_{k}+\frac{\Delta V}{\Delta t}, \\
& \dot{V}=\dot{z} \cdot A=\dot{z}_{k} \cdot A_{k}+\frac{\Delta V}{\Delta p} \cdot \frac{\Delta p}{\Delta t} .
\end{aligned}
$$

The following formula is used to define the volume flexibility $\kappa$ :

$$
\kappa=\frac{\Delta V}{\Delta p}
$$

Figure 2 presents a flow diagram of the inertial channel. When the upper chamber body receives a vertical excitation vibration, there is a pressure difference between the upper and lower cavities, which combines the change in the inertia channel displacement with the change in the volume flexibility.

$$
\begin{aligned}
& A \cdot \int \dot{z}=A_{k} \cdot \int \dot{z}_{k}+\kappa \cdot \int_{p_{u}}^{p_{0}} \frac{\partial p}{\partial t}, \\
& z \cdot A=z_{k} \cdot A_{k}+\kappa \cdot\left(p_{0}-p_{u}\right), \\
& p_{0}-p_{u}=\frac{z \cdot A}{\kappa}\left(1-\frac{z_{k}}{z} \cdot \frac{A_{k}}{A}\right) .
\end{aligned}
$$

The mass of the inertial channel is set as $m_{k}$. The transformation of the differential equation of motion is as follows:

$$
m_{k}=A_{k} \cdot L_{k} \cdot \rho
$$

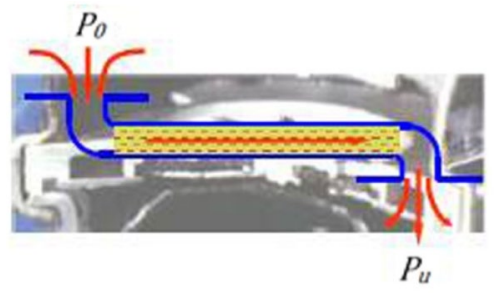

Figure 2 Inertial channel diagram 


$$
\begin{aligned}
& m_{k} \ddot{z}_{k}+k_{k} \cdot \dot{z}_{k}=A_{k}\left(p_{0}-p_{u}\right), \\
& m_{k} s^{2} z_{k}+k_{k} \cdot \mathrm{s} \cdot z_{k}=A_{k}\left(p_{0}-p_{u}\right), \\
& m_{k} s^{2} z_{k}+k_{k} \cdot \mathrm{s} \cdot z_{k}=A_{k} \cdot \frac{z \cdot A}{\kappa}\left(1-\frac{z_{k}}{z} \cdot \frac{A_{k}}{A}\right), \\
& \kappa \cdot m_{k} s^{2} \frac{z_{k}}{z}+\kappa \cdot k_{k} \cdot \mathrm{s} \cdot\left(\frac{z_{k}}{z}\right) \\
& =A_{k} \cdot A-A_{k} \cdot A \cdot\left(\frac{z_{k}}{z}\right) \cdot \frac{A_{k}}{A}, \\
& \frac{z_{k}}{z}=\frac{A}{A_{k}} \cdot \frac{\omega^{2}}{\omega_{k}^{2}+2 \zeta_{k} \omega_{k} \cdot j \omega-\omega^{2}},
\end{aligned}
$$

$\omega_{k}^{2}=\frac{A_{k}^{2}}{\kappa \bullet m_{k}}=\frac{c_{k}}{m_{k}} \zeta_{k}=\frac{k_{k}}{2 \sqrt{c_{k} m_{k}}}$, where $\omega_{k}$ is the fluid vibration element frequency of the inertial channel, $k_{k}$ is the fluid column stiffness of the inertial channel, $\zeta_{k}$ is the damping ratio, and $\zeta_{k}$ is a physical quantity that varies with the frequency and is related to the amplitude. The formula is derived as follows:

$$
\zeta_{c}=0.35 *\left(\left(2 \frac{A \delta X}{A_{c} l_{c}}\right)^{2}+\frac{\left(1-\lambda^{2}\right)^{4}}{\lambda^{4}}\right)^{1 / 4} .
$$

Based on Eqs. (2)-(4), the following equations can be obtained:

$$
\begin{aligned}
& C_{d y}(\omega)=k_{s}+c_{s} \cdot \omega+\frac{A^{2}}{\kappa} \cdot\left(1-\frac{z_{k}}{z} \cdot \frac{A_{k}}{A}\right) \\
& \left|C_{d y n}(\omega)\right|=\sqrt{\operatorname{Re}(\omega)^{2}+\operatorname{Im}(\omega)^{2}}, \\
& |\varphi(\omega)|=\tan \left(\frac{\operatorname{Im}(\omega)}{\operatorname{Re}(\omega)}\right)^{-1} .
\end{aligned}
$$

The above is the expression of the dynamic stiffness. To simplify the formula, the trend of the dynamic stiffness and loss angle is obtained. The dimensionless expression is introduced to make the data more convenient and the program converge faster.

$$
C_{d y n}(\omega)=\frac{C_{d y}(\omega)}{k_{s}}
$$

The following parameters are defined:

$$
\lambda=\frac{\omega}{\omega_{k}}, N=\frac{A^{2}}{k_{s} \kappa},
$$

where $\lambda$ is the frequency ratio and $N$ is the coefficient of the hydraulic mount. In general, the coefficient of a good hydraulic mount is approximately 0.5 to 0.8 .

$$
\begin{aligned}
& C_{d y n}(\lambda)=1+N \cdot\left(1-\frac{1}{\left(1-\lambda^{2}\right)}\right) \\
& \varphi(\lambda)=\tan \left(\frac{0}{\operatorname{Re}(\lambda)}\right)^{-1} .
\end{aligned}
$$

The above expression is first discussed, followed by the synthesized curves.

For feature point 1 , when the frequency ratio $\lambda$ is 0 , the following parameter is obtained:

$$
C_{d y n}(0)=1 ; \varphi(0)=0^{\circ} .
$$

For feature point 2, when the magnification factor $C_{d y n}$ is 0 , the following parameter is obtained:

$$
\begin{aligned}
& C_{d y n}(\lambda)=0, \\
& \lambda_{1,2}= \pm \frac{1}{\sqrt{1+N}} \varphi\left(\lambda_{1,2}\right)=\left\{\begin{array}{c}
0, \\
180^{\circ} .
\end{array}\right.
\end{aligned}
$$

For feature point 3 , when the frequency ratio $\lambda$ is 1 ; that is, at the resonance point, the following parameter is obtained:

$$
C_{d y n}(1)=\infty ; \varphi(0)=180^{\circ} .
$$

For feature point 4 , when the frequency ratio $\lambda$ is $+\infty$, the following parameter is obtained:

$$
\lim _{\lambda \rightarrow \infty} C_{d y n}(\lambda)=1+N ; \varphi(0)=0^{\circ} .
$$

The change rules of the dynamic stiffness and loss angle are depicted in Figures 3 and 4. The dynamic stiffness changes with an increasing frequency ratio, as follows: first a decrease, then an increase to the maximum value, and then a decrease. When the dynamic stiffness reaches

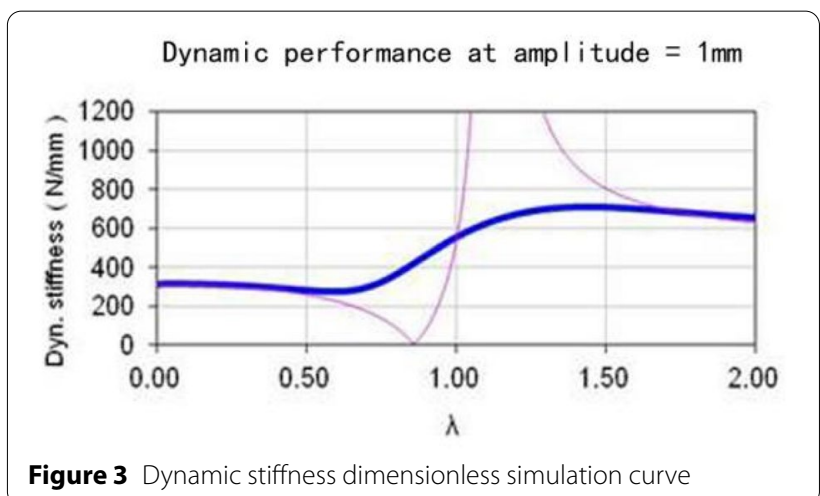




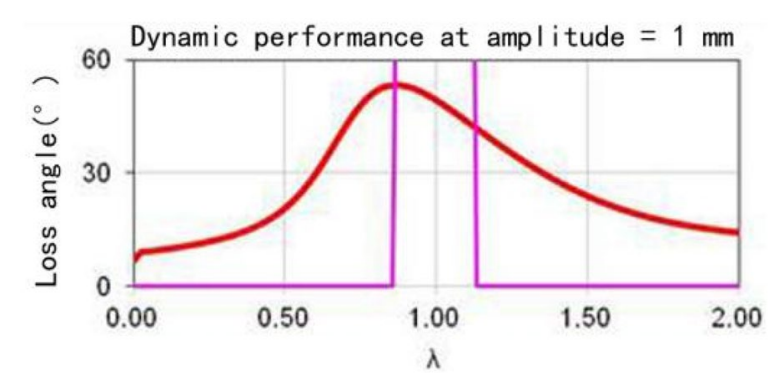

Figure 4 Loss angle dimensionless simulation curve

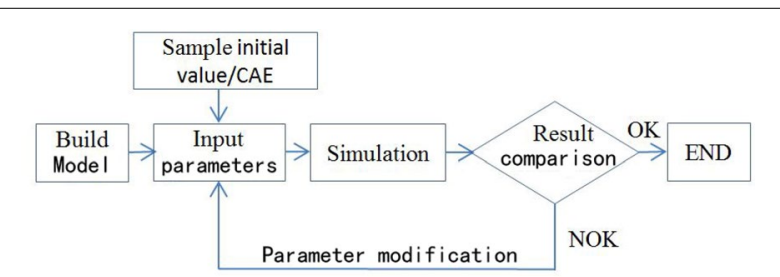

Figure 5 Parameter acquisition process

the minimum value, the maximum phase angle of the loss angle appears. After the resonance frequency, where $\lambda$ is 1 , the minimum value appears. The dynamic stiffness and loss angle can be clearly distinguished using this method.

\section{Simulation and Verification of Dynamic Characteristics}

\subsection{Acquired Parameters of Hydraulic Mount}

The main parameters of the hydraulic mount can be obtained by measurement and CAE analysis according to the data model. Through numerous of experimental validations and accumulations, this method can be conveniently used to guide the preliminary design and it can be corrected in subsequent tests, which plays a decisive role in the positive development of hydraulic mounts. The concept of the parameter design is shown in Figure 5.

The fluid density is obtained through the actual filling material, the area and length of the inertial passage are determined by measurement, and the equivalent piston area of the upper chamber $A$ can be obtained by the middle section.

$$
A=\frac{1}{2} \times\left(A_{1}+A_{2}\right)
$$

where $A_{1}$ and $A_{2}$ are the upper and lower sections of the upper chamber, respectively.

The calculation of the volume flexibility is relatively complicated because the rubber material is nonlinear. First, based on the general simplified principle in the analysis, it is assumed that the rubber material in each

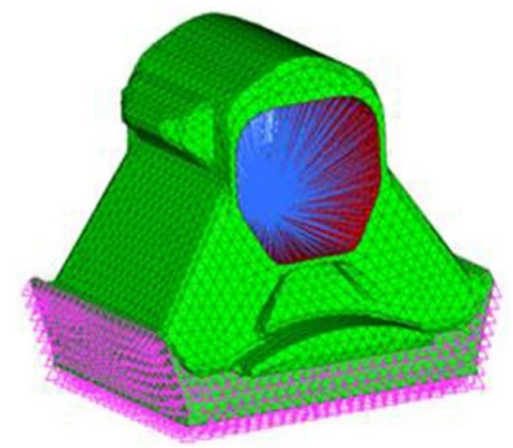

Figure 6 Finite elements of calculation grid model

Table 1 Key parameters of finite element analysis

\begin{tabular}{ll}
\hline Parameter & Value \\
\hline Elasticity modulus (MPa) & $7.3 \times 10^{4}$ \\
Poisson's ratio & 0.33 \\
Aluminum frame density $\left(\mathrm{kg} / \mathrm{mm}^{3}\right)$ & $2.7 \times 10^{-9}$ \\
Main spring hardness $\left(^{\circ}\right)$ & 48 \\
C01 & 0.07 \\
C10 & 0.27 \\
Grid nodes & 7179 \\
Grid elements & 31072 \\
\hline
\end{tabular}

direction is incompressible hyperelastic. Only the nonlinearity of the rubber is considered, whereas its viscoelasticity is excluded. In this case, the function expression of the Mooney-Rivlin material model is used for the calculation. The function is as follows:

$$
U=\sum_{i+j=1}^{N} C_{i j}\left(I_{1}-3\right)^{i}\left(I_{2}-3\right)^{j}+\sum_{i=1}^{N} \frac{1}{D}\left(\sqrt{I_{3}}-1\right)^{2 i} .
$$

Owing to the bidirectional decoupling diaphragm pressure, the deformation of the decoupling diaphragm is ignored. The decoupling diaphragm is simplified to an elastic sheet without holes, which is connected to the rubber of the main spring by a common node. The material is the same as that of the main spring. The connection between the bottom of the decoupled diaphragm, and the housing of the main spring and upper and lower cases is constrained. The calculation model is depicted in Figure 6.

When the volume and pressure changes of the upper chamber are known, the volume flexibility can be obtained by Eq. (2), where the key parameters required are listed in Table 1.

$$
C=\Delta V / \Delta P \text {. }
$$




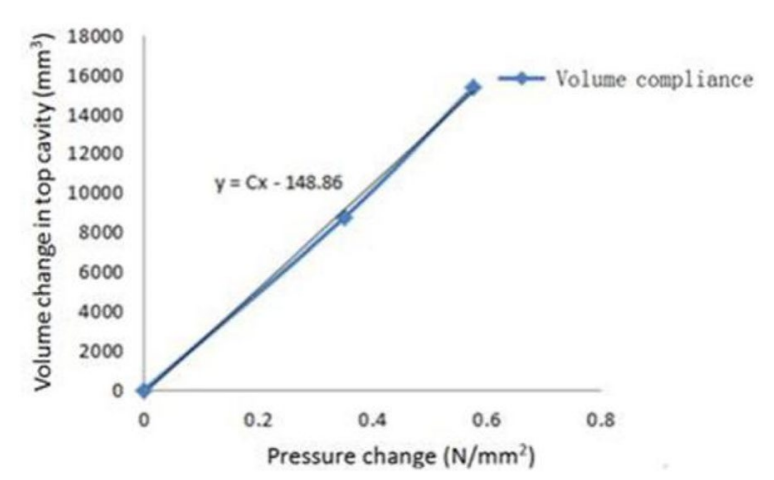

Figure 7 Volume compliance fitting curve

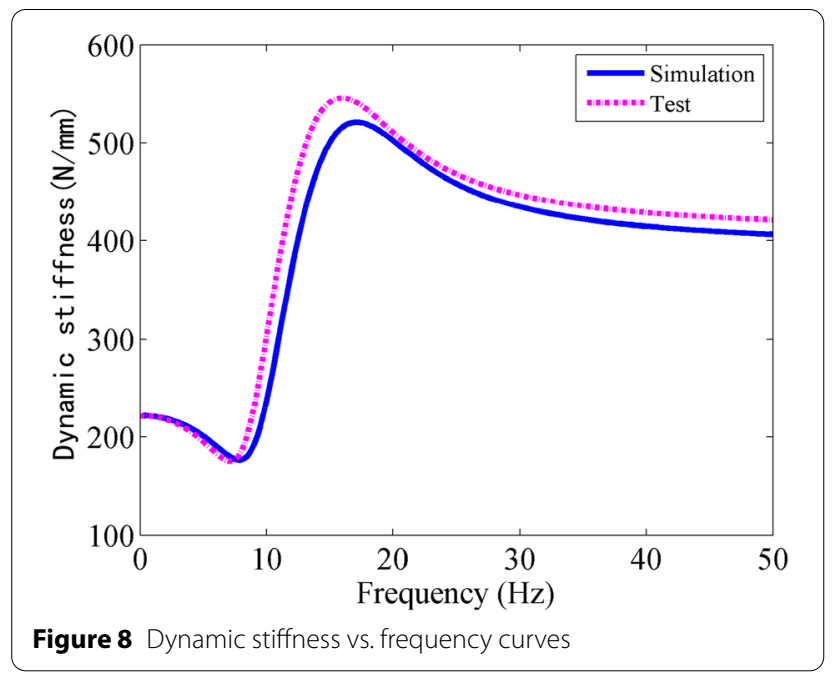

The fitting curve is presented in Figure 7. It should be noted that the lower cavity is free to deform and is connected to the outside world owing to its small thickness, which means that the fluctuation of the pressure is small and it is ignored here.

\subsection{Verification of Dynamic Simulation}

Hydraulic mounts can reflect unique features under lowfrequency, larges amplitudes [25-29]. With an amplitude of $1 \mathrm{~mm}$ as the excitation source, the variation laws of the dynamic stiffness and loss angle were investigated and compared with the measured data, as shown in Figures 8 and 9. The test curve was a real measurement result of the hydraulic mount, and the simulation curve was obtained from the simulation analysis.

It can be observed from Figures 8 and 9 that the difference between the experimental and simulation curves was small. The reason for this error is that the main spring was properly simplified, and its real state

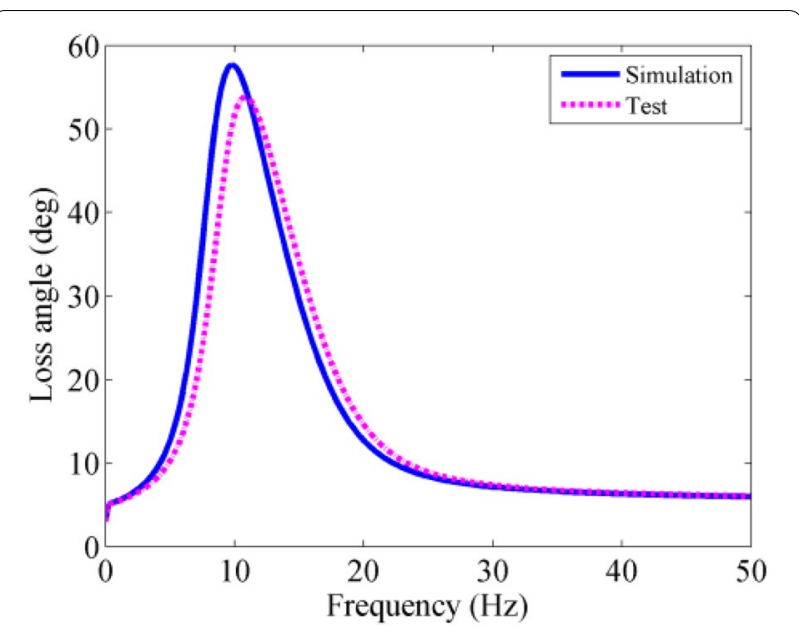

Figure 9 Loss angle vs. frequency curves

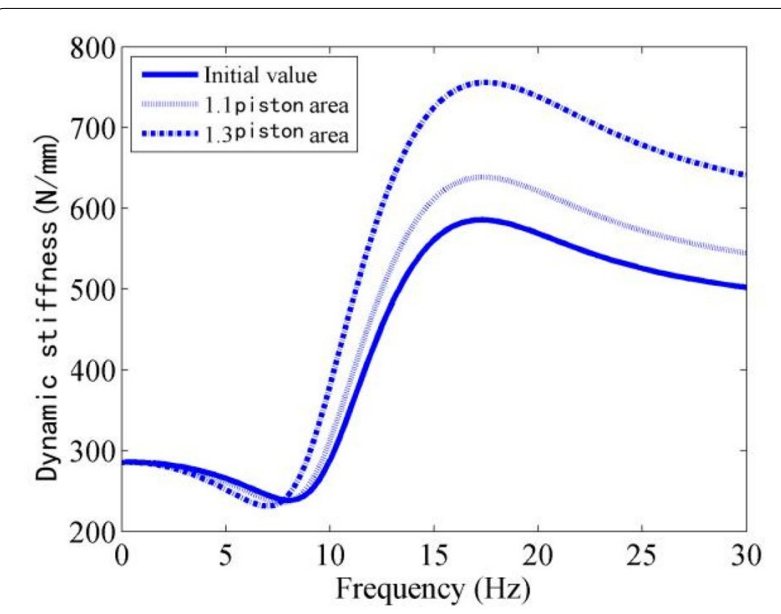

Figure 10 Dynamic stiffness vs. frequency curves in different piston areas

was strongly nonlinear, which could not be avoided in the simulation analysis. The relative error between $10 \%$ and $20 \%$ could meet the requirements of the project, and the accuracy of the simulation model was verified.

\section{Analysis of Influence of Parameter Variation on Hydraulic Performance}

\subsection{Influence of Equivalent Piston Area on Dynamic Characteristics}

As illustrated in Figures 10 and 11, the peaks of the dynamic stiffness and loss angle increased gradually with an increase in the equivalent piston area. However, the increase in the frequency was not obvious. 


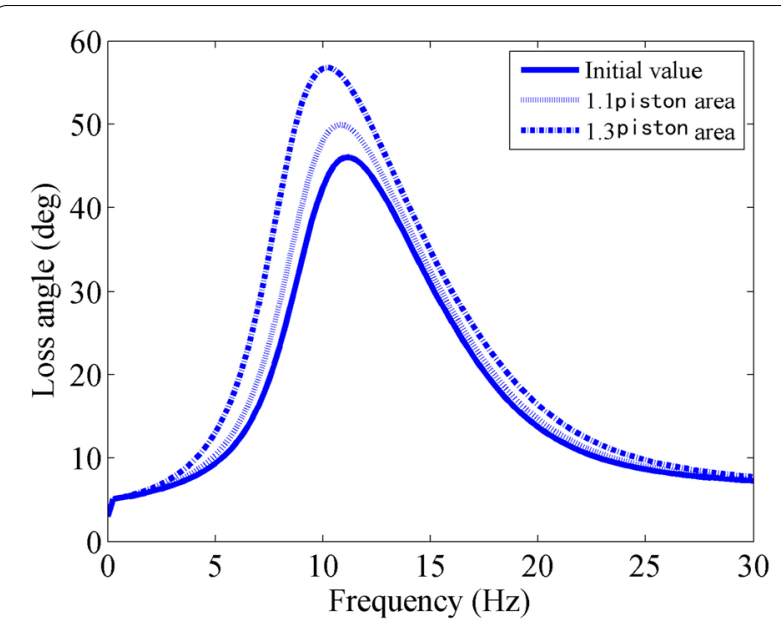

Figure 11 Loss angle vs. frequency curves in different piston areas

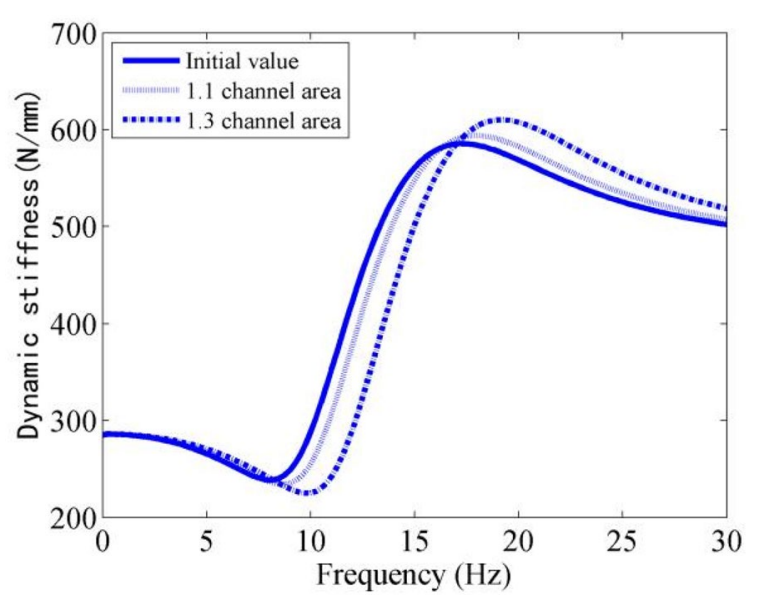

Figure 12 Dynamic stiffness vs. frequency curves in different inertial channel areas

\subsection{Influence of Liquid Column Length on Dynamic Characteristics}

As illustrated in Figures 12 and 13, as the cross-sectional area increased, the peaks of the dynamic stiffness and loss angle increased slightly. However, the peak frequency increase was more obvious, which was caused by the greater inertia effect.

\subsection{Influence of Liquid Column Length on Dynamic Characteristics}

As illustrated in Figures 14 and 15, as the length increased, the peaks of the dynamic stiffness and loss angle increased, but the frequency decreased continuously. This is because the increase in the length

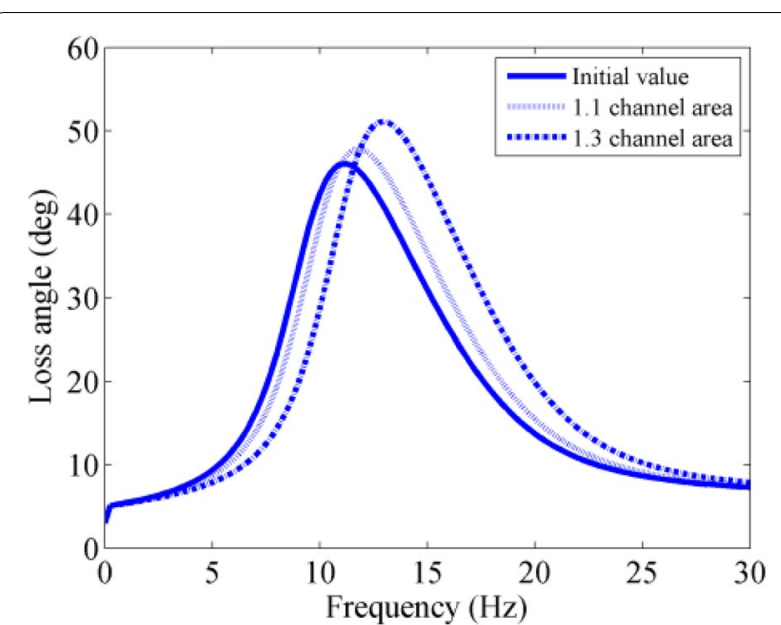

Figure 13 Loss angle vs. frequency curves in different inertial channel areas

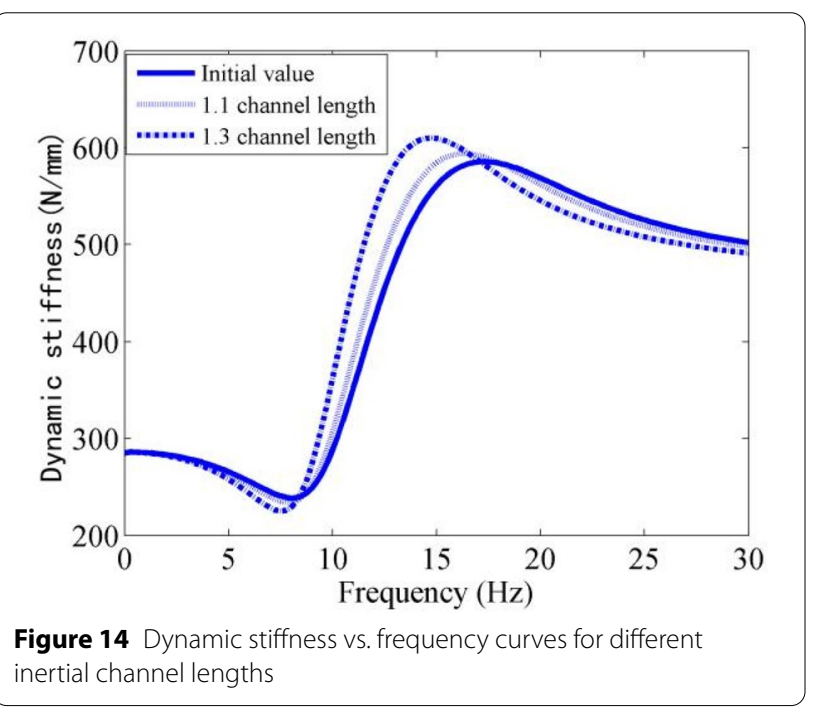

corresponded to an increase in the mass and a decrease in the fixed frequency.

\subsection{Influence of Volume Flexibility on Dynamic Characteristics}

As illustrated in Figures 16 and 17, the peaks of the dynamic stiffness and loss angle decreased with an increase in the volume flexibility, whereas the frequency decreased.

The change was owing to the increase in the volume flexibility, which means that the volume stiffness decreased and the motion displacement increased. In the case of a constant vibration source, the dynamic stiffness decreased and the loss angle changes followed the same rule. 


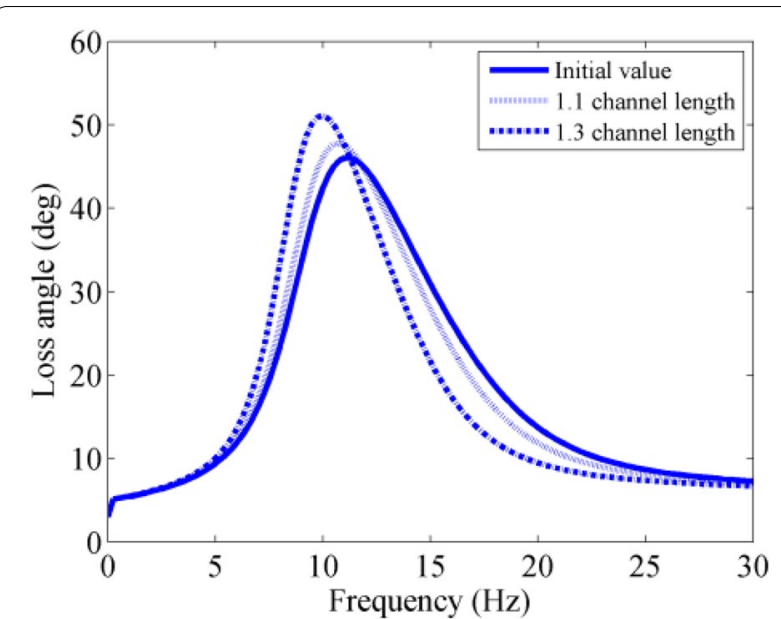

Figure 15 Loss angle vs. frequency curves for different inertial channel lengths

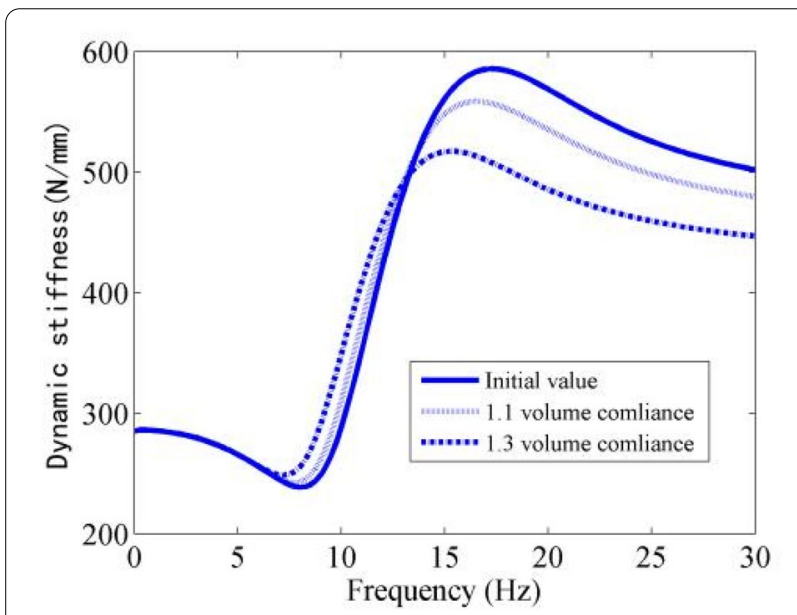

Figure 16 Dynamic stiffness vs. frequency curves for different volume compliances

According to the influence of the hydraulic mount parameters on its dynamic performance, the vibration of the dynamic stiffness and loss angle peaks can be controlled by optimizing the equivalent piston area. The peak and frequency can be optimized through the port area, length, and volume flexibility simultaneously.

\section{Application of Case}

To improve the driving experience and comfort, it was necessary to adjust the vibration characteristics of the hydraulic mount at a test site during the course of the project.

The test condition was to pass a specific road at a constant speed at the test site. The vertical vibration of the

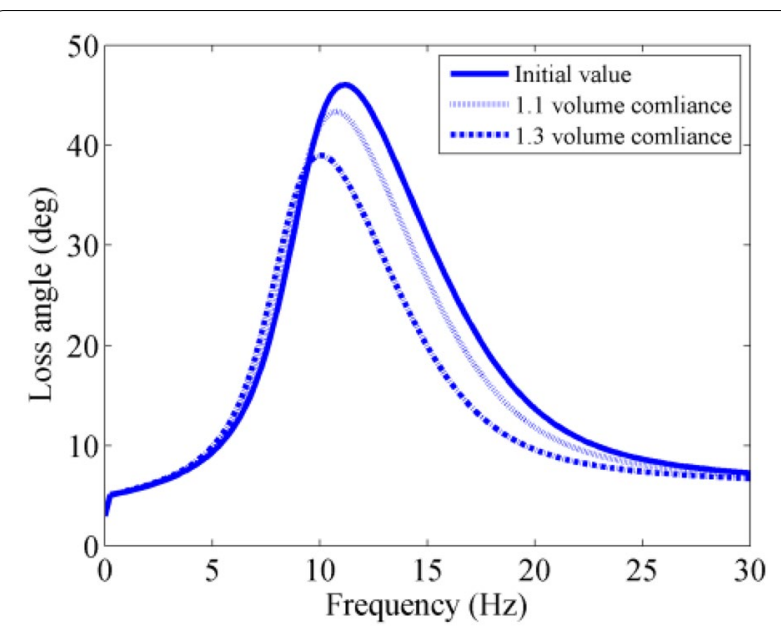

Figure 17 Loss angle vs. frequency curves for different volume compliances

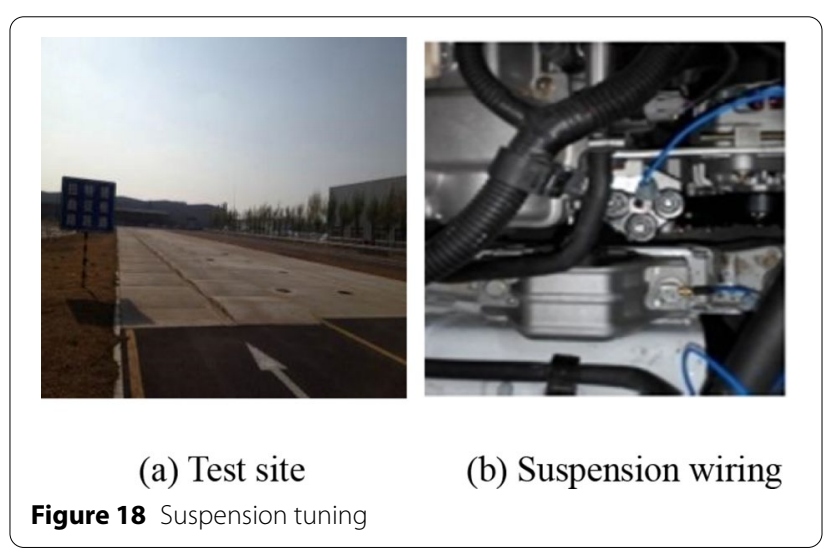

real vehicle was obvious and the driving performance was poor, as illustrated in Figure 18.

During the adjustment process, the PCB acceleration sensor, Devesoft data acquisition instrument, and other equipment were tested objectively. The results were optimized, as indicated in Figure 19. The peak vibration was large before optimization and was consistent with the subjective feeling. The structural parameters were modified and the sample test was executed again. The peak acceleration and peak frequency were significantly reduced. The optimized curve is presented in Figure 19.

The key parameters of the hydraulic mount are displayed in Table 2.

\section{Conclusions}

A mechanical model of a hydraulic mount was established by means of fluid mechanics and mechanical structures. The parameterization of the structural 


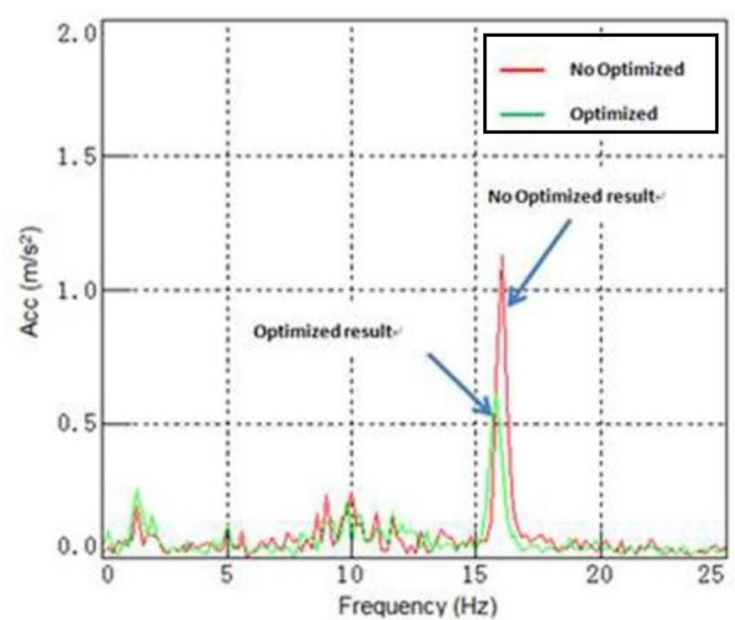

Figure 19 Acceleration vs. frequency curves

Table 2 Key parameters of hydraulic mount.

\begin{tabular}{ll}
\hline Parameter & Value \\
\hline Fluid density $\left(\mathrm{kg} / \mathrm{mm}^{3}\right)$ & $1.06 \times 10^{-6}$ \\
0 Hz stiffness $(\mathrm{N} / \mathrm{mm})$ & 311 \\
0 Hz loss angle $\left(^{\circ}\right)$ & 7 \\
Piston area $\left(\mathrm{mm}^{2}\right)$ & 3087 \\
Cavity area $\left(\mathrm{mm}^{2}\right)$ & 68.5 \\
Cavity length $(\mathrm{mm})$ & 254 \\
Loss factor & 2.86 \\
Volume compliance $\left(\mathrm{mm}^{5} / \mathrm{N}\right)$ & 43942 \\
\hline
\end{tabular}

variables and the establishment of dimensionless formulas were of significant help in the early stages of design. The specific research results demonstrate the following.

1. Based on the mechanical model, the dimensionless and concrete expressions of the dynamic stiffness and loss angle were derived, which could lay a solid foundation for the study of dynamic characteristics.

2. Through the impact analysis of the parameter variation, the structural parameters and frequency peaks were established with a one-to-one correspondence. This is of guiding significance for the matching of mounts.

3. Real vehicle tests demonstrated that the dynamic performance of liquid resistance can be improved by optimizing reasonable structural parameters, which may contribute to the overall performance of the vehicle, and reduce the research and development cycle.

\section{Acknowledgements}

Thanks very much for the State Key Laboratory of Automobile Simulation and Control to provide the test environment.

\section{Authors' Contributions}

$\mathrm{YL}$ is responsible for the whole paper writing and conducted the simulation model. XG gave the advice on the abstract. PL reviewed the introduction and RG checked the validation result. All authors read and approved the final manuscript.

\section{Authors' Information}

Yanhua Liu, born in 1973, is currently an engineer at Brilliance Auto R\&D Center, Shenyang, China. He received his master's degree from Jilin University, China, in 2011. His research interests include the chassis system and dynamic simulation.

Xin Guan, Doctor of Engineering, Distinguished Professor of Cheung Kong scholar, He is the president of Automobile Research Institute, Jilin University, China. The vice president of the Automobile Engineering Society of China, and the counselor of Jilin Provincial Government.

Pingping Lu, is a teacher at Jilin University, Changchun, China. She received her doctor from Jilin University, China, in 2012. Her search interests include the chassis system and dynamic simulation.

Rui Guo, is a teacher at Jilin University, China. She received her PhD degree from Jilin University, China, in 2009. Her search interests include the chassis system and dynamic simulation.

\section{Funding}

Not applicable.

\section{Competing Interests}

The authors declare that they have no competing interests.

Received: 27 June 2019 Revised: 10 March 2021 Accepted: 13 May 2021 Published online: 31 May 2021

\section{References}

[1] M Tiwari, H Adiguna, R Singh. Experimental characterization of a nonlinear hydraulic engine mount. Noise Control Engineering Journal, 2003, 51(1): 36-49.

[2] R N Jazar, M F Golnaraghi. Nonlinear modeling, experimental verification, and theoretical analysis of a hydraulic engine mount. JVC/Journal of Vibration and Control, 2002, 8(1): 87-116.

[3] W B Shangguan, Z H Lu. Experimental study and simulation of a hydraulic engine mount with fully coupled fluid — structure interaction finite element analysis model. Computers and Structures, 2004, 82(22): 1751-1771.

[4] W Hi, W Yang, S Hou, et al. Experiment and simulation study on parameter obtaining of hydraulic mount with air spring. Advanced Materials Research, 2013, 710(9): 277-280.

[5] B H Qi, D Y Huang. Experimental analysis on dynamic characteristics of hydraulic mount. Machinery Design \& Manufacture, 2010, 7: 21-23.

[6] Z H Lv, W B Shangguan, W Liang, et al. Experimental methods and test evaluation for the dynamic characteristics of hydraulically damped rubber mount. China Mechanical Engineering, 2004, 15(2): 182-186. (in (hinese)

[7] L J Zhao, Z Zhou, W H Zeng, et al. Stiffness simulation analysis and experimental verification of hydraulic mount rubber. Mechanical Engineer, 2016, 5: 61-63.

[8] M Jiang, S Hou, J J Cai, et al. Simulation and experiment study for the dynamic characteristics of automobile engine hydraulic mount based on the key chart theory. Machinery Design \& Manufacture, 2008,04:70-72.

[9] K Jahani, M Dehnad. Identifying the frequency dependent material property of a hydraulic engine mount through an iterative procedure using 3D finite element modeling. Journal of Mechanical Science \& Technology 2014, 28(6): 2041-2047. 
[10] Z H Lv, W B Shangguan, W Liang. Experimental method and analysis of dynamic characteristics of liquid resistance rubber isolator. Chinese Mechanical Engineering, 2004, 15(2): 182-186. (in Chinese)

[11] W K Shi, Y Mao, X Jiang, et al. Parameter identification and dynamic characteristics of semi-active hydraulic mount. Journal of Jilin University, 2014, 44(3): 605-611.

[12] W B Shangguan, Z H Lu. Modelling of a hydraulic engine mount with fluid - structure interaction finite element analysis. Journal of Sound and Vibration, 2004, 275(1-2): 193-221.

[13] A Ster, S Hartmann, E Rank. p-FEM applied to finite isotropic hyperelastic bodies. Journal of Computational Methods in Applied Mechanics and Engineering, 2003, 192(47-48): 5147-5166.

[14] W W Chen, J R Wang, XX Chen, et al. Dynamic characteristic simulation of hydraulic engine mount based on ALE finite element method. Journal of Hefei University of Technology (Natural Science Edition), 2011, 34(005): 641-645.

[15] W K Shi, Y Mao, X Jiang, et al. Study on dynamic characteristics and parameter influence of engine's semi-active hydraulic mount using AMESim. Journal of Xi'an Jiaotong University, 2014, 48(1): 42-47+59.

[16] Y Yang. Research on adaptive cruise control systems and performance analysis using Matlab and Carsim. 2017 th International Conference on Mechanical, Automotive and Materials Engineering (CMAME); 2017.

[17] W B Shangguan, Z H Lv. Simulation analysis of nonlinear characteristics of liquid resistance rubber isolator. Journal of Vibration Engineering, 2003, 16(4): 393-398.

[18] M Niazkar, S H Afzali. Analysis of water distribution networks using MAT$L A B$ and Excel spreadsheet: h-based methods. Computer Applications in Engineering Education, 2017, 25(1): 129-141.

[19] M Sulaiman, H M Hussein, R Omar, et al. Dynamic stability analysis of generator with power system stabilizers using Matlab Simulink. Indonesian Journal of Electrical Engineering \& Computer Science, 2016, 2(3): 501.
[20] S J Hou, W Yang. Dynamic characteristics and parametric sensitivity of engine air spring hydraulic mount. Journal of Vibration and Shock, 2019, 21: 178-185.

[21] R L Fan, X L Zhang. Study on semi-active hydraulic mount with variablestiffness decoupling membrane. Journal of Mechanical Engineering, 2015, 51(14): 108-114. (in Chinese)

[22] J L Xie, H H Li. Analysis of dynamic characteristics of hydraulic excavator working device. Mechanical Engineering \& Automation, 2016, 02: 63-65.

[23] D Y Pan, X Gao, Z Zhu, et al. Exploring dynamic characteristics of hydraulic engine mount with decoupler using a switch system. Mechanical Science and Technology for Aerospace Engineering, 2016, 7: 1120-1124.

[24] X Han, YT Pan. Study on dynamic characteristics and exploitation of engine semi-active hydraulic mount. Machinery Design \& Manufacture, 2010, 4: 134-135

[25] Z J Xu, B B Zhang, Y B Sun, et al. Dynamic characteristic of hydraulic mount with inertia channel and optimal design. Chinese Internal Combustion Engine Engineering, 2002, 5: 27-30+33.

[26] D Y Huang, C S Zhang, H J Shen. Analysis and research on dynamic characteristics of hydraulic mount. Tractor \& Farm Transporter, 2009, 3: $26-28+31$.

[27] D M Jiao, M J Zheng, JW Chen. Analysis of dynamic characteristics and vibration isolation performance of hydraulic engine mount. Noise and Vibration Control, 2011, 6: 127-130.

[28] Y W Lee, CW Lee. Dynamic analysis and control of an active engine mount system. Automobile Engineering, 2002, 216: 921-931.

[29] L Jiang, Z Zhu, H Liu, et al. Analysis of dynamic characteristics of water hydraulic rotating angle self-servo robot joint actuator. Journal of Intelligent \& Robotic Systems, 2018, 2(5): 1118-1136.

\section{Submit your manuscript to a SpringerOpen ${ }^{\odot}$ journal and benefit from:}

- Convenient online submission

- Rigorous peer review

- Open access: articles freely available online

- High visibility within the field

- Retaining the copyright to your article

Submit your next manuscript at $\boldsymbol{\nabla}$ springeropen.com 